\title{
On microprocessor-based computers
}

\author{
E. JAMES KEHOE, LLOYD J. FREI, ROBERT W. TAIT, and I. GORMEZANO \\ University of Iowa, Iowa City, Iowa 52242
}

\begin{abstract}
Economical microprocessor-based computers have been recently developed by Motorola and Control Logic, Inc. Apparently, in response to the price competition, DEC has introduced the PDP-8/A, incorporating the memory characteristics of the microprocessor-based computers. Relative to older minicomputers, the microprocessor-based systems and the PDP-8/A "miniprocessor" require more time to execute an experimentally useful task. However, their real-time operating characteristics would appear to be sufficient for on-line use in behavioral control and data acquisition. The PDP-8/A's compatibility with older models of the PDP-8 series enables it to use previously developed software, but, for purposes of software development, the microprocessor systems of fer a more powerful architecture and instruction set.
\end{abstract}

The introduction of microprocessors would appear to offer a relatively inexpensive means for applying computer technology to the acquisition and control of behavioral data. However, as noted by McLean (1974), microprocessors, since they are only central processing units (CPUs), require considerable additional circuitry to fulfill memory, timing, and $\mathrm{I} / \mathrm{O}$ functions to interact with the experimental environment. Yet, in the brief period since McLean's observation, at least two microprocessor-based systems, with the necessary support logic and memory, have become available. Motorola incorporated its M6800 microprocessor into a complete system, the Motorola Exorciser, while Intel's 8080 microprocessor has been incorporated into a computer system by Control Logic, Inc. To meet this competition, DEC has introduced the PDP-8/A, their "miniprocessor," as a completely packaged system which can be directly interfaced to peripheral devices. As yet, there has not been an assessment of the relative merits of these systems in behavioral applications. To provide a preliminary basis for such assessment, these systems were examined on the basis of the following criteria: cost of a minimal system, availability of software support, ease of software programming, and capability of real-time operation. Through so considering the merits of these three systems, the present paper is intended to contribute to an understanding of the applicability of microprocessors to behavioral investigations.

Two distinct characteristics have emerged in the development of microprocessor computer systems. First, in contrast to the 12- and 16-bit word lengths of currently employed minicomputers, Motorola's M6800 and Intel's 8080 microprocessors employ 8-bit word lengths. Although DEC markets an 8-bit microprocessor (M7341), the PDP-8/A employs a 12-bit processor, constructed of TTL logic chips, to maintain compatibility with the PDP-8 minicomputer series.

The preparation of this report was supported by NSF Grant GB-41531.
However, the 8-bit word length found in Motorola's 8600 and Intel's 8080 microprocessors, would not place a serious limitation on the use of microprocessor systems for control and data acquisition, since a large number of peripheral devices employ 8-bit codes. Moreover, principally by the use of multiple-word instructions, 8-bit microprocessors achieve substantial flexibility in memory addressing. Second, in contrast to the conditions prevailing at the time of McLean's observations in 1973, advances in large scale integrated (LSI) circuitry have permitted the construction of microprocessor-based computer systems with less than 24 chips, thus markedly reducing maintenance requirements. Furthermore, the level of technical competence required for such maintenance would not appear to exceed that possessed by users of solid state logic modules (e.g., BRS).

In designing these computers, the manufacturers have provided the potential for the user to tailor the memory configuration to the minimum required for his task, and thus minimize his cost. As constructed, the units have the potential of extensively utilizing less expensive read-only memory (ROM) in lieu of random-access memory (RAM). By analogy with the core memory typical of minicomputers, ROM is permanently programmed memory employed for the storage of relatively invariant programs (e.g., subroutines for the servicing of peripheral devices). Since the contents of ROM cannot be modified in the course of experimental execution, variables used by the operating programs are stored in RAM. By the use of ROM, the size of RAM can be smaller than generally found in minicomputers. Furthermore, the major use of these systems, as envisaged here, is for control and data acquisition in behavioral investigations with statistical analyses being best left to larger computer facilities. Accordingly, the total RAM requirements would be relatively small, since the data would be rapidly transmitted to mass storage devices (e.g., punched paper tape, digital magnetic tape, or disk ). 


\section{COST OF A MINIMAL SYSTEM}

Each of the three computer systems under consideration can be purchased from their respective manufacturers with a minimal configuration containing the following in common: an enclosure with a bus oriented structure, console card, CPU, solid state memory, power supplies, TTY interface, and basic software. However, they differ in memory configuration, bus capacity, number of $1 / 0$ lines, and presence of an ROM field programmer. DEC's PDP-8/A offers $4 \mathrm{~K}$ of RAM, 12 I/O lines, an 8-slot bus, but no ROM field programmer. Whereas, Motorola's Exorciser contains $.75 \mathrm{~K}$ of RAM and $3 \mathrm{~K}$ of ROM, $16 \mathrm{I} / 0$ lines, a 14-slot bus, but no ROM field programmer. In turn, Control Logic's system provides $4 \mathrm{~K}$ of RAM, $12 \mathrm{I} / \mathrm{O}$ lines, a 16-slot bus, and an ROM field programmer. These basic systems may be purchased from the Digital Equipment Corporation (Maynard, Massachusetts 01754), Motorola (Semiconductor Products, Box 20912, Phoenix, Arizona 85036) and Control Logic Inc. ( 9 Tech Circle, Natick, Massachusetts 01760 ) for $\$ 2,600, \$ 2,700$, and $\$ 3,475$, respectively. For the specified price, one obtains turn-key systems requiring only the addition of interfaces (e.g., relay drivers) to the peripheral devices.

Substantial cost differences materialize in the further expansion of these minimal systems. Consider, for example, the expansion of each of these systems by the addition of basic units of RAM and $\mathrm{I} / \mathrm{O}$ lines. For $2 \mathrm{~K}$ of RAM, DEC, Motorola, and Control Logic ask \$960, $\$ 650$, and $\$ 650$, respectively; whereas, per $\mathrm{I} / \mathrm{O}$ line, DEC, Motorola, and Control Logic ask $\$ 42, \$ 15$, and $\$ 15$, respectively. Presently, ROM can be expanded only in the systems offered by DEC and Control Logic. For $2 \mathrm{~K}$ of reprogrammable ROM, Control Logic enjoys a substantial price advantage, charging only $\$ 520$, while DEC charges $\$ 960$. In addition, DEC's configuration requires the purchase, for $\$ 500$, of a memory extension board for any expansion beyond the basic $4 \mathrm{~K}$ of memory. Furthermore, Control Logic's system, containing an ROM field programmer, allows greater flexibility and speed in the alteration of reprogrammable ROMs. In the absence of an ROM field programmer unit, reprogramming must be accomplished by returning the ROM to the manufacturer or distributor.

\section{EVALUATION OF THE CPUS}

\section{Ease of Software Programming}

Basic software necessary for loading, editing, assembling, and debugging programs for RAM and ROM can be purchased from all three firms. Beyond these fundamental programs, the PDP-8/A has available to it the vast body of programs developed for previous models of DEC's PDP-8 series. However, to accommodate these programs, the memory capacity required would normally exceed the $4 \mathrm{~K}$ of the basic system. Accordingly, to operate within the constraints of $4 \mathrm{~K}$ of memory, a substantial investment in software development may be required, for example, SKED (cf. Snapper, 1973). Although Motorola and Intel have announced formation of users groups, there is presently no readily available software appropriate for behavioral research. For users willing to invest the time necessary to develop their own software, Motorola's M6800 architecture and instruction set would appear to offer the greatest ease of programming, followed by Intel's 8080 , and trailing a distant third, the processor in DEC's PDP-8/A.

The programming capabilities of Motorola's 8-bit M6800 CPU approximate those found in 16-bit CPUs (e.g., Data General's NOVA, DEC's PDP-11). The instruction set of the M6800 contains many instructions using two and three words of memory, thus producing 16- and 24-bit instructions, respectively. The three-word instructions permit absolute addressing of 65,000 locations in memory whereas, the two-word instructions permit relative addressing of 125 locations on either side of any given location. Further economy and flexibility in addressing is achieved in the $M 6800$ by the availability of a 16-bit index register, which permits a two-word instruction to reference any location whose address value is up to 256 greater than the value contained in the index register. In addition to the index register, other architectural features of the M6800 include two accumulators, aiding manipulation of data, and a self-adjusting "stack" pointer, aiding the sequential storage and retrieval of data lists. Since many peripheral devices employ BCD codes, the Motorola M6800 provides instructions for arithmetic manipulation of two 4-bit BCD codes without converting the codes to binary form. For ease of decision making, condition-code registers enable two instructions to execute a conditional branch (i.e., jump or jump-to-subroutine) on the basis of a comparison between the contents of an accumulator and a memory location, without altering the contents of the accumulator. In addition to the programming features of the M6800 per se, a further aid to development of software is provided by standard routines contained within the 3K ROM of Motorola's Exorciser. This set of routines performs tasks commonly required in all laboratory control systems (e.g., TTY servicing, code conversion, and interrupt servicing).

In contrast to the programming features of Motorola's M6800, Intel's 8080 CPU provides less flexible and efficient addressing capabilities and fewer special registers. Although its instruction set contains two- and three-word instructions, a relative addressing capability is not available. Accordingly, the dominant mode for referencing memory locations would appear to be absolute addressing, thus requiring space consuming three-word instructions. To provide some flexibility in addressing, a 16-bit "index" register is available in Intel's 8080. However, it would appear to be somewhat 
cumbersome to employ since its contents, in addition to being fixed by specific instructions, are altered by the relative value designated in any instruction using the index register. For purposes of data manipulation, storage, and retrieval, Intel's 8080 possesses a single accumulator, a "stack" pointer, and BCD arithmetic capabilities. Comparable to the decision making capabilities of Motorola's M6800, the instruction set of Intel's 8080 enables a comparison and a conditional branch to be made with two instructions. 1

The programming capabilities of Intel's 8080 and, clearly, Motorola's M6800, far exceed those of the CPU found in the PDP-8/A. As best as can be discerned, the programming features of the PDP-8/A are identical to those of the PDP-8/E. Specifically, the PDP-8/A's instruction set contains only single-word 12-bit instructions, and the addressing scheme is limited to DEC's singular blend of direct and indirect addressing as constrained by the division of memory in to "pages" of 128 locations. Specifically, it is possible for a single instruction to directly address only the 128 locations on the page containing the particular instruction and the first 128 locations in memory, referred to as Page " 0 ." Hence, indirect addressing, involving two words of memory, is required to reference any other location in memory. For manipulation, storage, and retrieval of data, the PDP-8/A offers a single accumulator and "autoindex" registers, which automatically increment whenever indirectly addressed. In contrast to the two-instruction compare-and-branch operations available in the Motorola M6800 and Intel 8080, 7 to 10 instructions would be required to perform a similar operation in the PDP-8/A. DEC appears to have done an admirable job in developing as powerful a programming capability as can be realized with a single word, 12-bit instruction length. However, the 8-bit microprocessors under consideration, through their use of multiple-word instructions, have been able to mimic the power of programming inherent in processors employing 16- or 24-bit word lengths.

\section{Real-Time Operations}

The feasibility of using the systems under consideration for real-time control and data acquisition depends on the time necessary to perform an experimentally useful task, which is a product of execution time for a single instruction and the number of executed instructions. To provide a basis for general assessment, as well as for comparison among these systems, two routines that would generally be found in experimental control programs were written for each of these systems. These routines were designed to serve basic stimulus control and response counting functions in a clock-driven operating system. Specifically, the first routine was designed to count response inputs on any or all of eight response input lines from an experimental station. The second routine was designed to control the presentation and duration of a state-dependent sequence of stimulus patterns transmitted on eight separate output lines. For each routine, the three systems were compared on the basis of the time required to service a single station with eight input and eight output lines. In writing these routines, an earnest attempt was made to optimize their efficiency. However, we realize that a more efficient algorithm may have been overlooked.

For purposes of comparison among systems, it was assumed that response or clock-pulse counting requirements would not exceed 4,095. To attain this capabilitiy with Motorola's M6800 and Intel's 8080 microprocessors, it was necessary to provide a double-precision algorithm for both the response-counter and stimulus-control routines. While the algorithm applied to these microprocessor routines acquired the capacity to accommodate counts of approximately 65,000 , the utilization of double-precision did not affect the relative standing of each of the CPUs in execution time. In calculating execution times for the response-counter routine, the extreme condition was assumed in which data appeared simultaneously on all eight input lines. Similarly, for the stimulus-control routine, it was assumed that a change in stimulus pattern would occur on all eight output lines. Under these extreme conditions, all three CPUs using RAM would be able to execute both response-counter and stimulus-control routines for one station in less than $1 \mathrm{msec}(\mathrm{PDP}-8 / \mathrm{A}=342 \mathrm{microsec}$, $\mathrm{M} 6800=422$ microsec, and $8080=484$ microsec). Of course, servicing each comparable additional station requires the addition of the respective single-station service times.

\section{DISCUSSION}

While the application of minicomputers to behavioral research has substantially increased the capabilities for complex real-time control and data acquisition, the "miniprocessor" and microprocessor computer systems do not appear to offer any major advances along these lines. Rather, they appear to represent primarily a contribution to the economy of establishing a computer-based laboratory. At the present time, two economies would appear realizable. First, the cost of initial systems is substantially less than that of comparable minicomputers. For example, the PDP-8/A system, as previously described, costs approximately $50 \%$ less than a PDP-8/E of comparable configuration. However, while the initial cost of microprocessor-based systems is similar to that of the PDP-8/A, their further expansion through the use of LSI components is less expensive than comparable expansion of the PDP-8/A with its older integrated circuitry. Second, in microprocessor systems, the appreciably smaller number of chips employed relative to minicomputers, should decease maintenance costs through reducing the 
probability of failure and time necessary to identify faulty components.

In exchange for the above economies, the systems considered have sacrificed some speed and certain memory characteristics. The PDP-8/E, for example, requires at least $40 \%$ less time than the PDP-8/A or microprocessor systems to execute the routines used to evaluate their respective real-time capabilities. With regard to memory characteristics, the contents of currently available RAM are "volatile" in that power to memory must be maintained in order to retain programs and data in storage. On the other hand, the contents of ROM may be retained without power, but at a cost of being relatively inflexible. However, in the foreseeable future, these limitations of speed and memory appear rectifiable. Currently, the technology exists for the mating of microprocessors and memory chips which would yield microprocessor-based computer systems with speed capabilities exceeding those of present minicomputers. Prototypes of "nonvolatile" RAM, that mimic magnetic core memory, are currently being tested in engineering laboratories (e.g., Nitron Corp., 10420 Bubb Road, Cupertino, California 95014). Accordingly, realistic prospects exist for the appearance of microprocessor systems with the speed and memory characteristics of minicomputers, but with the same economies found in current microprocessor systems.
Conceivably, further reduction in the cost of establishing a computer controlled laboratory could be realized by facilities with sufficient technical personnel to design the relatively simple circuit boards necessary to accommodate the less than two dozen chips found in current microprocessor systems. After assembly, such circuit boards would be installed in prewired bus oriented enclosures that are available from present manufacturers of microprocessor-based systems. Accordingly, for laboratories or entire departments, the fabrication of microprocessor systems in this fashion would permit the economical development of multiple, but individually tailored, microprocessor systems containing varying quantities of ROM, RAM, and $\mathrm{I} / \mathrm{O}$ options.

\section{REFERENCES}

McLean, R. S. Microcomputers for experimental psychology. Behavior Research Methods \& Instrumentation, 1974, 6, 155-158.

Snapper, A. G. Use of a notation system for digital control and recording. Behavior Research Methods \& Instrumentation, $1973,5,124-128$.

\section{NOTE}

1. For further information on Intel's 8080 , write to Intel Corporation, 3065 Bowers Avenue, Santa Clara, California 95051 . 